\section{Interação dos fatores sociodemográficos na associação entre fatores psicossociais e transporte ativo para a escola}

\author{
Interaction of sociodemographic factors in the \\ association between psychosocial factors and \\ active commuting to school
}

Edina Maria de Camargo 1

Maria Paula Maia Santos 2

Anelize Gabriele Peressute Ribeiro 1

Jorge Mota 2

Wagner de Campos 1 doi: 10.1590/0102-311X00102719

\author{
Correspondência \\ E. M. Camargo \\ Universidade Federal do Paraná. \\ Rua Coração de Maria 92, Curitiba, PR 80210-132, Brasil. \\ edinacamargo@gmail.com \\ 1 Universidade Federal do Paraná, Curitiba, Brasil. \\ 2 Faculdade de Desporto, Universidade do Porto, Porto, Portugal.
}

pais e nível socioeconômico. O apoio social e a autoeficácia foram mensurados por questionários. Caminhar, pedalar ou usar skate foram considerados meios de transporte ativo para a escola. A regressão logística binária foi utilizada para verificar a associação dos fatores psicossociais com o transporte ativo para a escola. Em seguida, termos de interação com as variáveis sociodemográficas (idade, escolaridade pai, escolaridade mãe, nível socioeconômico) foram incluídos na relação dos fatores psicossociais com o transporte ativo para a escola. A amostra foi composta de 1984 adolescentes (55,9\% feminino). Desses, 748 (37,7\% da amostra) relataram utilizar transporte ativo para a escola em uma semana habitual. As análises de interação demonstraram que escolaridade e nível socioeconômico alto estão associados negativamente com transporte ativo para escola. Nivel socioeconômico baixo e escolaridade baixa estão associados positivamente. A escala de apoio social dos amigos apresentou um número maior de variáveis significativas quando comparada às demais escalas. Os resultados indicam que os fatores sociodemográficos (escolaridade dos pais e nível socioeconômico) moderam a associação dos fatores psicossociais com o transporte ativo para a escola.

Atividade Motora; Adolescentes; Apoio Social; Autoeficácia 


\section{Introdução}

Níveis insuficientes de atividade física estão aumentando em muitos países, com grandes implicações para o aumento da prevalência de doenças não transmissíveis e para a saúde geral da população 1,2,3,4,5. $\mathrm{Na}$ Europa, o estudo HELENA (Healthy Lifestyle in Europe by Nutrition in Adolescence) 6, realizado em nove países (Grécia, Alemanha, Bélgica, França, Hungria, Itália, Suécia, Áustria e Espanha), mostrou um grande número de adolescentes ( $80,1 \%$ do sexo feminino e $46,3 \%$ do masculino) que não cumprem as recomendações de sessenta minutos ou mais de atividade física diária ${ }^{7}$. Ainda nessa linha, o Health Behavior in School-Aged Children (HBSC) 8 realizado em 48 países, na Europa e América do Norte, indicou que $86 \%$ das meninas e $71 \%$ dos meninos não cumprem a recomendação de atividade física. Além disso, o estudo ANIBES (Estudio de Antropometría, Ingesta y Balance Energético en España) ${ }^{9}$, realizado na Espanha com crianças na faixa etária entre 9 e 12 anos, relatou uma prevalência de 39\% para o sexo masculino e $62 \%$ para o feminino como insuficientemente ativos e, para adolescentes entre 13 e 17 anos, os dados apontaram $50 \%$ e $86 \%$, para meninos e meninas, respectivamente 9 .

No Brasil, dados de estudos sobre a prevalência da atividade física disponíveis até o momento indicam que crianças e adolescentes não cumprem as recomendações mínimas 10,11,12. O estudo ERICA (Estudos dos Riscos Cardiovasculares em Adolescentes) indica que 54,3\% (70,7\% feminino e 38\% masculino) dos adolescentes brasileiros não cumprem as recomendações de 300 minutos ou mais por semana de atividade física 13 .

O transporte ativo para a escola é uma maneira eficaz de incorporar atividade física às atividades diárias dos adolescentes e aumentar os níveis gerais de atividade física 14 . A curta distância (até $3 \mathrm{~km}$ a pé e até $5 \mathrm{~km}$ de bicicleta/skate) tem sido mencionada como um dos preditores mais consistentes em estudos com adolescentes 14,15,16,17,18. No entanto, mesmo para destinos dentro de uma distância viável 14,15,16,17,18, os adolescentes mais velhos usam regularmente modos de transporte passivos para a escola, como carro e motocicleta 14,15,16,17,18. Isso indica que existe a necessidade de maiores esforços para aumentar o transporte ativo para a escola em adolescentes mais velhos 14,19. Embora os adolescentes nem sempre morem a uma distância viável da escola, é possível que existam destinos propensos ao transporte ativo para a escola 14,19. Estudos que investiguem variáveis associadas ao transporte ativo para a escola e as possíveis variáveis de interação podem ampliar a compreensão sobre o tema.

Modelos ecológicos afirmam que o comportamento ativo é influenciado por vários fatores em múltiplos níveis, incluindo fatores psicossociais (como apoio social para atividade física, autoeficácia para atividade física) e ambientais (como segurança, calçadas) 4,20,21. Além disso, esses fatores podem variar de acordo com os fatores sociodemográficos (como sexo, idade, escolaridade dos pais e nível socioeconômico) 4,20,21. Evidências sobre a relação entre os fatores psicossociais (como apoio social de pais e amigos e autoeficácia) e transporte ativo para a escola podem ser importantes do ponto de vista de maximizar níveis de atividade em adolescentes 14,15,16,17,18,19,21. Acrescenta-se que investigar a interação 22 dos fatores sociodemográficos (como idade, escolaridade dos pais e nível socioeconômico) ainda não investigados nos estudos anteriores pode ajudar a compreender melhor a associação dos fatores psicossociais com o transporte ativo para a escola, uma vez que fatores sociodemográficos parecem moderar os resultados.

Sendo assim, o objetivo do estudo foi investigar a interação dos fatores sociodemográficos na associação dos fatores psicossociais com o transporte ativo para a escola.

\section{Métodos}

Trata-se de um estudo com delineamento transversal, realizado no ano de 2018, com amostra representativa de adolescentes, de 15 a 17 anos de idade, matriculados no Ensino Médio da rede estadual da cidade de Curitiba, Paraná, Brasil. Para o cálculo amostral, a priori, foi considerada uma associação de RP (razão de prevalência) = 1,49 entre o apoio social e a prática de atividades físicas 23 , uma prevalência de insuficientemente ativos de $50 \% 24$, um nível de $95 \%$ de confiança $(\alpha=0,05)$ com um poder de $80 \%(\beta=0,20)$, resultando em uma amostra mínima de 804 sujeitos. Entretanto, foi considerado um acréscimo de $30 \%$ para possíveis perdas e recusas, o que estimou uma amostra mínima necessária para o estudo de 965 sujeitos. 
A amostra foi selecionada a partir do processo de amostragem por estágios múltiplos, conglomerados, da seguinte forma: 1o estágio - todas as escolas estaduais foram estratificadas de acordo com as nove regiões administrativas do Município de Curitiba e foi realizado um sorteio de duas escolas em cada uma das regiões administrativas da cidade; 2o estágio - foi realizada uma seleção aleatória simples de uma turma de cada ano do Ensino Médio, de acordo com a quantidade de escolares, necessária para determinada região administrativa do município. Por fim, todos os alunos de cada turma foram convidados a participar do estudo.

No total, foram avaliados 2.506 adolescentes, porém foram excluídos aqueles que apresentaram limitações físicas e/ou cognitivas que limitassem a prática de atividade física (informado pelo escolar, $\mathrm{n}=12)$ e aqueles que tivessem 18 anos $(\mathrm{n}=125)$. Os adolescentes que não apresentaram o Termo de Consentimento Livre e Esclarecido assinado pelos pais ou responsáveis $(\mathrm{n}=104)$, aqueles que se negaram a participar do estudo ou faltaram no dia da coleta $(n=56)$ e os que responderam os questionários incorretamente $(\mathrm{n}=229)$ foram considerados como perda amostral. Assim, a amostra analítica do estudo foi de 1.984 adolescentes.

Análises estatísticas realizadas a posteriori mostraram que essa amostra poderia identificar RP estatisticamente significantes acima de 1,28 como risco e abaixo de 0,77 como fator de proteção, considerando um poder de $80 \%$ ( $\beta=20 \%$ ), um nível de $95 \%$ de confiança $(\alpha=5 \%)$ e considerando uma prevalência de $34 \%$ de adolescentes com baixo apoio social e que não realizam transporte ativo para a escola.

\section{Fatores sociodemográficos}

O sexo foi autorreportado (masculino, feminino). Por sua vez, a idade foi calculada a partir da data de nascimento (informada pelo adolescente), subtraída da data de coleta dos dados e dividida por 365, posteriormente sendo classificada em: 15 anos, 16 anos, 17 anos. A escolaridade dos pais e responsáveis e também o nível socioeconômico foram avaliados segundo os Critérios de Classificação Econômica da Associação Brasileira de Empresas de Pesquisa (ABEP. http://www.abep.org/criteriobrasil). A escolaridade dos pais foi classificada em: até Ensino Fundamental completo, Ensino Médio completo e Ensino Superior completo (foi perguntado sobre a escolaridade do pai e da mãe). Para o cálculo do nível socioeconômico, foi utilizada a escolaridade do chefe da família (os adolescentes deveriam reportar quem era o chefe da família e sua escolaridade). Por fim, o nível socioeconômico foi classificado em três categorias: "baixo" (classe C+D), "médio" (classe B1+B2) e "alto" (classe A1+A2) (ABEP, http://www.abep.org/criterio-brasil).

Os fatores sociodemográficos (idade, escolaridade do pai, escolaridade da mãe e nível socioeconômico) foram testados nas análises de interação (multiplicação da possível variável moderadora pela variável independente - exemplo: idade*apoio social dos pais-praticar).

\section{Fatores psicossociais}

\section{- Apoio social para a prática de atividade física}

O apoio social de pais e amigos para a prática de atividade física foi mensurado por meio de uma escala de 10 itens, escala ASAFA (Escala de Apoio Social para Prática de Atividade Física para Adolescentes), que apresenta consistência interna satisfatória [pais: $\alpha \geq 0,77$ e IFC (índice de fidedignidade combinada) $\geq 0,83$; amigos: $\alpha \geq 0,87$ e IFC $\geq 0,91] 25$. Os adolescentes informaram a frequência (nunca $=1$, raramente $=2$, frequentemente $=3$, sempre $=4$ ) com que pais e amigos ofereciam algum tipo de apoio social para a prática de atividade física (incentivam, praticam, transportam, assistem, comentam, convidam) durante uma semana típica ou normal 25. Para efeitos de análise, e com o objetivo de garantir comparabilidade com estudos semelhantes ${ }^{26}$, as opções de resposta "raramente" e "frequentemente" foram agrupadas e classificadas como "às vezes". Assim, as categorias de frequência semanal de apoio social foram classificadas como "nunca", "às vezes" e "sempre". O escore geral de apoio social de pais e amigos foi computado (somatório dos valores atribuídos a cada item - 5 a 20 pontos) e categorizado pela mediana: baixo apoio social e elevado apoio social. 


\section{- Autoeficácia para atividade física}

A percepção de autoeficácia para a prática de atividade física foi mensurada por meio de uma escala composta de dez itens (consistência interna $\alpha=0,81$ e reprodutibilidade - coeficiente de correlação intraclasse CCI = 0,67; IC95\%: 0,58-0,75) 27 que considera o quanto os adolescentes se percebem capazes de praticar atividade física na presença de barreiras. Os itens foram ancorados por uma escala Likert de quatro pontos (discordo muito $=1$, discordo $=2$, concordo $=3$, concordo muito $=4$ ).

Para efeitos de análise, e com o objetivo de garantir comparabilidade com estudos semelhantes 28 , as opções de resposta "discordo muito e discordo" e "concordo e concordo muito" foram agrupadas e classificadas como "discordo" e "concordo". O escore geral de autoeficácia foi computado (somatório dos valores atribuídos a cada item - 10 a 40 pontos) e categorizado pela mediana: "baixa autoeficácia" e "elevada autoeficácia”.

\section{- Transporte ativo para a escola}

Foi avaliado o modo de transporte usado para ir e voltar da escola durante uma semana típica, por meio da questão: "Em uma semana escolar normal, quantos dias e quanto tempo por dia você usa os seguintes meios de deslocamento (caminhada, bicicleta, skate, ônibus, transporte escolar, carro)?”. Todos os estudantes que relataram caminhar, andar de bicicleta ou usar skate para ir e voltar da escola foram classificados como "transporte ativo para a escola"; os demais, como "passivos" (ônibus, van e carro). Essa questão mostrou confiabilidade teste-reteste adequada (IC95\%: 0,90-0,95; p < 0,05) e tem sido utilizada em estudos semelhantes 29,30,31.

\section{Análise dos dados}

Para evitar viés relacionado ao processo de seleção amostral com característica complexa (estratificação por conglomerado), as análises de associação foram corrigidas pelo delineamento complexo, utilizando o comando complex sample do software SPSS versão 23.0 (https://www.ibm.com/). Foi utilizada regressão logística binária de forma bruta e ajustada para verificar a associação dos fatores psicossociais com o transporte ativo para a escola, sendo modelo 1: AS (apoio social) pais; modelo 2: AS amigos; modelo 3: AE (autoeficácia). Permaneceram na análise ajustada somente as variáveis que apresentaram valores de $\mathrm{p} \leq 0,20$ na análise bruta. Todas as análises foram realizadas separadamente para cada sexo, e foi adotado um nível de $5 \%$ de significância.

Em seguida, foram testadas as interações dos fatores sociodemográficos (idade, escolaridade do pai, escolaridade da mãe, nível socioeconômico) na relação AS pais x TAE (transporte ativo para a escola); AS amigos x TAE; AE x TAE. Para isso, foram introduzidos termos de interação nos modelos de análises da regressão logística (multiplicação da possível variável moderadora pela variável independente - exemplo: idade*AS dos amigos: "praticam"). Ao todo, 92 interações foram testadas (as quatro variáveis sociodemográficas foram testadas com todas as 12 variáveis da escala de apoio social e as 11 variáveis da escala de autoeficácia). As interações significativas $(\mathrm{p}<0,05)$ foram apresentadas em figuras.

\section{Comitê de ética}

O estudo seguiu as normas de pesquisa envolvendo seres humanos do Conselho Nacional de Saúde (Resolução no 466/2012), sendo aprovado pelo Comitê de Ética em Pesquisa da Universidade Federal do Paraná (CAAE: 98133218.8.0000.0102) e autorizado pelos pais ou responsáveis dos adolescentes por meio do Termo de Consentimento Livre e Esclarecido. 


\section{Resultados}

A amostra foi composta de 1.984 adolescentes (sendo 55,9\% do sexo feminino). Desses, somente 748 (37,7\% da amostra) relataram utilizar transporte ativo para a escola em uma semana habitual. Em relação ao sexo, dos 748 adolescentes que relataram utilizar o transporte ativo para a escola, 57\% (426) são meninas e $43 \%$ (322) são meninos. Adolescentes mais velhos $(17$ anos $=30.5)$ utilizam menos o transporte ativo para a escola quando comparados aos mais novos (15 e 16 anos =69,5\%). Adolescentes que têm pais com escolaridade até Ensino Médio completo utilizam mais transporte ativo para a escola $(76,7 \%)$ quando comparados a adolescentes que têm pais com Ensino Superior completo. Quando verificado o nível socieconômico, $78,6 \%$ da amostra que utilizam o transporte ativo para a escola pertencem às classes $\mathrm{B} e \mathrm{C}$.

As associações, bruta e ajustada, entre os fatores psicossociais com o transporte ativo para a escola para meninos podem ser verificadas na Tabela 1. Com relação ao apoio social dos pais, os resultados apresentados na análise ajustada identificaram que meninos cujos pais incentivam a prática de atividade física foram menos propensos a realizar o transporte ativo ("às vezes": RC = 0,64; IC95\%: 0,42-0,96 e "sempre": RC = 0,58; IC95\%: 0,36-0,95). Por outro lado, meninos cujos pais praticam "às vezes" atividade física juntos ( $\mathrm{RC}=1,57$; IC95\%: 1,09-2,25) e que oferecem "sempre" transporte para que realizem atividade física ( $\mathrm{RC}=1,56$; IC95\%: 1,04-2,32), assim como pais que comentam "sempre" que eles estão fazendo bem a atividade física ( $R C=1,73$; IC95\%: 1,08-2,76), foram associados positivamente com o transporte ativo para a escola. O escore de pais foi associado positivamente com o transporte ativo para a escola $(\mathrm{RC}=1,37$; IC95\%: 1,04-1,81).

\section{Tabela 1}

Associação bruta e ajustada dos fatores psicossociais com o transporte ativo para a escola em meninos $(n=875)$.

\begin{tabular}{|c|c|c|c|c|c|c|}
\hline & \multirow[t]{2}{*}{$\mathbf{n}$} & \multirow[t]{2}{*}{$\%$} & \multicolumn{2}{|c|}{ Bruta } & \multicolumn{2}{|c|}{ Ajustada * } \\
\hline & & & $\mathrm{RC}$ & IC95\% & RC & IC95\% \\
\hline \multicolumn{7}{|c|}{ Apoio social pais } \\
\hline \multicolumn{7}{|l|}{ Incentivam } \\
\hline Nunca & 67 & 38,7 & 1,00 & & 1,00 & \\
\hline Às vezes & 169 & 43,4 & 0,67 & $0,45-1,00$ & 0,64 & $0,42-0,96$ ** \\
\hline Sempre & 86 & 46,2 & 0,60 & $0,37-0,98$ & 0,58 & $0,36-0,95$ ** \\
\hline \multicolumn{7}{|l|}{ Praticam } \\
\hline Nunca & 99 & 35,7 & 1,00 & & 1,00 & \\
\hline Às vezes & 178 & 46,8 & 1,49 & $1,05-2,10$ & 1,57 & $1,09-2,25 * *$ \\
\hline Sempre & 45 & 49,5 & 1,19 & $0,70-2,03$ & 1,23 & $0,72-2,09$ \\
\hline \multicolumn{7}{|c|}{ Transportam } \\
\hline Nunca & 140 & 38,5 & 1,00 & & 1,00 & \\
\hline Às vezes & 95 & 42,4 & 1,06 & $0,75-1,51$ & 1,03 & $0,72-1,48$ \\
\hline Sempre & 87 & 54,4 & 1,64 & $1,11-2,41$ & 1,56 & $1,04-2,32$ ** \\
\hline \multicolumn{7}{|l|}{ Assistem } \\
\hline Nunca & 130 & 40,5 & 1,00 & & & \\
\hline Às vezes & 139 & 43,6 & 0,83 & $0,57-1,21$ & & \\
\hline Sempre & 53 & 49,1 & 0,74 & $0,44-1,23$ & & \\
\hline \multicolumn{7}{|l|}{ Comentam } \\
\hline Nunca & 99 & 36,4 & 1,00 & & 1,00 & \\
\hline Às vezes & 134 & 47,5 & 1,55 & $1,04-2,32$ & 1,39 & $0,93-2,05$ \\
\hline Sempre & 89 & 45,9 & 1,72 & $1,09-2,73$ & 1,73 & $1,08-2,76$ ** \\
\hline
\end{tabular}

(continua) 
Tabela 1 (continuação)

\begin{tabular}{|c|c|c|c|c|c|c|}
\hline & \multirow[t]{2}{*}{$\mathrm{n}$} & \multirow[t]{2}{*}{$\%$} & \multicolumn{2}{|c|}{ Bruta } & \multicolumn{2}{|c|}{ Ajustada * } \\
\hline & & & RC & IC95\% & RC & IC95\% \\
\hline \multicolumn{7}{|c|}{ Escore do apoio social pais } \\
\hline Baixo & 481 & 55,0 & 1,00 & & 1,00 & \\
\hline Elevado & 394 & 45,0 & 1,44 & $1,06-1,96$ & 1,37 & $1,04-1,81$ ** \\
\hline \multicolumn{7}{|c|}{ Apoio social amigos } \\
\hline \multicolumn{7}{|l|}{ Incentivam } \\
\hline Nunca & 117 & 40,1 & 1,00 & & 1,00 & \\
\hline Às vezes & 137 & 46,3 & 0,93 & $0,64-1,34$ & 0,94 & $0,65-1,36$ \\
\hline Sempre & 68 & 42,5 & 0,65 & $0,40-1,06$ & 0,68 & $0,42-1,12$ \\
\hline \multicolumn{7}{|l|}{ Praticam } \\
\hline Nunca & 70 & 34,8 & 1,00 & & 1,00 & \\
\hline Às vezes & 140 & 43,5 & 1,54 & $1,00-2,39$ & 1,50 & $0,97-2,30$ \\
\hline Sempre & 112 & 49,8 & 1,97 & $1,16-3,33$ & 2,23 & $1,35-3,69 * * *$ \\
\hline \multicolumn{7}{|l|}{ Convidam } \\
\hline Nunca & 81 & 33,8 & 1,00 & & & \\
\hline Às vezes & 138 & 46,8 & 1,03 & $0,65-1,58$ & & \\
\hline Sempre & 103 & 48,4 & 1,40 & $0,82-2,38$ & & \\
\hline \multicolumn{7}{|l|}{ Assistem } \\
\hline Nunca & 157 & 43,7 & 1,00 & & 1,00 & \\
\hline Às vezes & 115 & 42,4 & 0,70 & $0,47-1,04$ & 0,63 & $0,42-0,95$ ** \\
\hline Sempre & 50 & 42,4 & 0,53 & $0,30-0,93$ & 0,45 & $0,25-0,79 * * *$ \\
\hline \multicolumn{7}{|l|}{ Comentam } \\
\hline Nunca & 146 & 39,7 & 1,00 & & 1,00 & \\
\hline Às vezes & 122 & 46,7 & 1,30 & $0,90-1,88$ & 1,15 & $0,78-1,70$ \\
\hline Sempre & 54 & 45,4 & 1,31 & $0,78-2,19$ & 1,14 & $0,66-1,97$ \\
\hline \multicolumn{7}{|c|}{ Escore do apoio social amigos } \\
\hline Baixo & 451 & 42,4 & 1,00 & & & \\
\hline Elevado & 424 & 48,5 & 0,89 & $0,66-1,21$ & & \\
\hline \multicolumn{7}{|c|}{ Autoeficácia - mesmos que... } \\
\hline \multicolumn{7}{|c|}{...esteja cansado, estressado } \\
\hline Discordo & 188 & 21,5 & 1,00 & & & \\
\hline Concordo & 687 & 78,5 & 1,19 & $0,82-1,71$ & & \\
\hline \multicolumn{7}{|c|}{...tenha outras coisas } \\
\hline \multicolumn{7}{|c|}{ interessantes para fazer } \\
\hline Discordo & 243 & 27,8 & 1,00 & & & \\
\hline Concordo & 632 & 72,2 & 1,05 & $0,75-1,46$ & & \\
\hline \multicolumn{7}{|c|}{...não tenha ninguém para ir } \\
\hline \multicolumn{7}{|l|}{ comigo } \\
\hline Discordo & 251 & 28,7 & 1,00 & & & \\
\hline Concordo & 624 & 71,3 & 0,92 & $0,67-1,27$ & & \\
\hline \multicolumn{7}{|c|}{...amigos convidem para fazer } \\
\hline \multicolumn{7}{|l|}{ outras coisas } \\
\hline Discordo & 341 & 39,0 & 1,00 & & 1,00 & \\
\hline Concordo & 534 & 61,0 & 1,50 & $1,11-2,03$ & 1,48 & $1,10-1,97 * * *$ \\
\hline \multicolumn{7}{|c|}{...pudesse assistir TV, jogar } \\
\hline videogames, $\iota$ & & & & & & \\
\hline Discordo & 483 & 55,2 & 1,00 & & & \\
\hline Concordo & 392 & 44,8 & 0,92 & $0,67-1,27$ & & \\
\hline
\end{tabular}

(continua) 


\begin{tabular}{|c|c|c|c|c|c|c|}
\hline & \multirow[t]{2}{*}{$\mathbf{n}$} & \multirow[t]{2}{*}{$\%$} & \multicolumn{2}{|c|}{ Bruta } & \multicolumn{2}{|c|}{ Ajustada * } \\
\hline & & & RC & IC95\% & RC & IC95\% \\
\hline \multicolumn{7}{|c|}{$\begin{array}{l}\text {...esteja sem vontade, } \\
\text { desmotivado }\end{array}$} \\
\hline Discordo & 548 & 62,6 & 1,00 & & 1,00 & \\
\hline Concordo & 327 & 37,4 & 0,80 & $0,58-1,11$ & 0,78 & $0,58-1,05$ \\
\hline \multicolumn{7}{|c|}{$\begin{array}{l}\text {...tenha que pagar taxa, } \\
\text { mensalidade }\end{array}$} \\
\hline Discordo & 469 & 53,6 & 1,00 & & & \\
\hline Concordo & 406 & 46,4 & 0,91 & $0,67-1,23$ & & \\
\hline \multicolumn{7}{|c|}{...ache que não tenha habilidade } \\
\hline Discordo & 243 & 27,8 & 1,00 & & & \\
\hline Concordo & 632 & 72,2 & 1,15 & $0,83-1,61$ & & \\
\hline \multicolumn{7}{|c|}{...não tenham locais próximos a } \\
\hline \multicolumn{7}{|l|}{ minha casa } \\
\hline Discordo & 273 & 31,2 & 1,00 & & & \\
\hline Concordo & 602 & 68,8 & 0,96 & $0,69-1,33$ & & \\
\hline \multicolumn{7}{|c|}{...não tenha ninguém para } \\
\hline \multicolumn{7}{|l|}{ orientar } \\
\hline Discordo & 283 & 32,3 & 1,00 & & & \\
\hline Concordo & 592 & 67,7 & 0,96 & $0,69-1,33$ & & \\
\hline \multicolumn{7}{|c|}{ Escore da autoeficácia } \\
\hline Baixa & 261 & 29,8 & 1,00 & & & \\
\hline Elevada & 614 & 70,2 & 1,10 & $0,81-1,49$ & & \\
\hline
\end{tabular}

IC95\%: intervalo de 95\% de confiança; RC: razão de chance.

* As variáveis com $p<0,20$ foram inseridas novamente no modelo;

$* * p<0,05$;

$\star \star \star p<0,01$.

Com relação ao apoio social dos amigos, meninos que indicaram possuir amigos que praticam atividade física "sempre" apresentaram mais chances de realizar o transporte ativo para a escola $(\mathrm{RC}=$ 2,23; IC95\%: 1,35-3,69). Adicionalmente, meninos que possuem amigos que os assistem realizando atividade física foram menos propensos a realizar o transporte ativo para a escola ( $\mathrm{RC}=0,63$; IC95\%: 0,42-0,95 e RC = 0,45; IC95\%: 0,25-0,79). Na autoeficácia, apenas a variável “...mesmo que amigos convidem para fazer outras coisas" foi associada ao transporte ativo para a escola (RC = 1,48; IC95\%: $1,10-1,97)$.

A Tabela 2 apresenta as associações entre os fatores psicossociais e o transporte ativo para a escola para meninas. Apenas variáveis relacionadas ao apoio social provenientes dos amigos permaneceram associadas no modelo ajustado, indicando que meninas que possuem amigos que praticam "às vezes" atividade física junto com elas possuem mais chances de realizar o transporte ativo para a escola, quando comparadas às meninas que nunca recebem esse tipo de apoio (RC = 1,48; IC95\%: 1,04-2,10). Em contrapartida, meninas que possuem amigos que as convidam para fazerem atividade física "às vezes" foram menos propensas a realizar o transporte ativo para a escola (RC = 0,65; IC95\%: 0,46-0,93).

Na autoeficácia, as variáveis "mesmo que não tenha ninguém para ir comigo" ( $\mathrm{RC}=0,73$; IC95\%: 0,56-0,95) e "mesmo que tenha que pagar mensalidade" ( RC = 1,30; IC95\%: 1,00-1,69) foram associada ao transporte ativo para a escola. 
Tabela 2

Associação bruta e ajustada dos fatores psicossociais com o transporte ativo para a escola em meninas ( $n=1.109)$.

\begin{tabular}{|c|c|c|c|c|c|c|}
\hline & \multirow[t]{2}{*}{$\mathbf{n}$} & \multirow[t]{2}{*}{$\%$} & \multicolumn{2}{|c|}{ Bruta } & \multicolumn{2}{|c|}{ Ajustada * } \\
\hline & & & RC & IC95\% & RC & IC95\% \\
\hline \multicolumn{7}{|c|}{ Apoio social pais } \\
\hline \multicolumn{7}{|l|}{ Incentivam } \\
\hline Nunca & 106 & 61,3 & 1,00 & & 1,00 & \\
\hline Às vezes & 220 & 56,6 & 0,70 & $0,50-0,98$ & 0,81 & $0,59-1,12$ \\
\hline Sempre & 100 & 53,8 & 0,80 & $0,52-1,23$ & 0,90 & $0,60-1,33$ \\
\hline \multicolumn{7}{|l|}{ Praticam } \\
\hline Nunca & 178 & 64,3 & 1,00 & & & \\
\hline Às vezes & 202 & 53,2 & 1,04 & $0,77-1,40$ & & \\
\hline Sempre & 46 & 50,5 & 1,03 & $0,63-1,66$ & & \\
\hline \multicolumn{7}{|c|}{ Transportam } \\
\hline Nunca & 224 & 61,5 & 1,00 & & 1,00 & \\
\hline Às vezes & 129 & 57,6 & 0,79 & $0,58-1,07$ & 0,86 & $0,64-1,15$ \\
\hline Sempre & 73 & 45,6 & 0,71 & $0,48-1,04$ & 0,75 & $0,52-1,08$ \\
\hline \multicolumn{7}{|l|}{ Assistem } \\
\hline Nunca & 191 & 59,5 & 1,00 & & & \\
\hline Às vezes & 180 & 56,4 & 1,20 & $0,87-1,66$ & & \\
\hline Sempre & 55 & 50,9 & 0,98 & $0,62-1,54$ & & \\
\hline \multicolumn{7}{|l|}{ Comentam } \\
\hline Nunca & 173 & 63,6 & 1,00 & & & \\
\hline Às vezes & 148 & 52,5 & 1,18 & $0,84-1,66$ & & \\
\hline Sempre & 105 & 54,1 & 1,08 & $0,72-1,61$ & & \\
\hline \multicolumn{7}{|c|}{ Escore do apoio social pais } \\
\hline Baixo & 640 & 57,7 & 1,00 & & & \\
\hline Elevado & 469 & 42,3 & 8,84 & $0,64-1,10$ & & \\
\hline \multicolumn{7}{|c|}{ Apoio social amigos } \\
\hline \multicolumn{7}{|l|}{ Incentivam } \\
\hline Nunca & 175 & 59,9 & 1,00 & & & \\
\hline Às vezes & 159 & 53,7 & 0,98 & $0,70-1,37$ & & \\
\hline Sempre & 92 & 57,5 & 1,09 & $0,72-1,66$ & & \\
\hline \multicolumn{7}{|l|}{ Praticam } \\
\hline Nunca & 131 & 65,2 & 1,00 & & 1,00 & \\
\hline Às vezes & 182 & 56,5 & 1,39 & $0,96-2,01$ & 1,48 & $1,04-2,10$ ** \\
\hline Sempre & 113 & 50,2 & 1,24 & $0,79-1,95$ & 1,35 & $0,88-2,07$ \\
\hline \multicolumn{7}{|l|}{ Convidam } \\
\hline Nunca & 159 & 66,3 & 1,00 & & 1,00 & \\
\hline Às vezes & 157 & 53,2 & 0,62 & $0,43-0,91$ & 0,65 & $0,46-0,93 * *$ \\
\hline Sempre & 110 & 51,2 & 0,71 & $0,45-1,14$ & 0,73 & $0,48-1,11$ \\
\hline \multicolumn{7}{|l|}{ Assistem } \\
\hline Nunca & 202 & 56,3 & 1,00 & & & \\
\hline Às vezes & 156 & 57,6 & 1,00 & $0,72-1,40$ & & \\
\hline Sempre & 68 & 57,6 & 1,02 & $0,64-1,63$ & & \\
\hline \multicolumn{7}{|l|}{ Comentam } \\
\hline Nunca & 222 & 60,3 & 1,00 & & & \\
\hline Às vezes & 139 & 53,3 & 1,05 & $0,75-1,48$ & & \\
\hline Sempre & 65 & 54,6 & 0,90 & $0,56-1,43$ & & \\
\hline
\end{tabular}

(continua) 
Tabela 2 (continuação)

\begin{tabular}{|c|c|c|c|c|c|c|}
\hline & \multirow[t]{2}{*}{$\mathbf{n}$} & \multirow[t]{2}{*}{$\%$} & \multicolumn{2}{|c|}{ Bruta } & \multicolumn{2}{|c|}{ Ajustada * } \\
\hline & & & RC & IC95\% & RC & IC95\% \\
\hline \multicolumn{7}{|c|}{ Escore do apoio social amigos } \\
\hline Baixo & 628 & 56,6 & 1,00 & & & \\
\hline Elevado & 481 & 43,4 & 0,95 & $0,73-1,24$ & & \\
\hline \multicolumn{7}{|c|}{ Autoeficácia - mesmos que... } \\
\hline \multicolumn{7}{|c|}{...esteja cansado, estressado } \\
\hline Discordo & 393 & 35,4 & 1,00 & & & \\
\hline Concordo & 716 & 64,6 & 0,96 & $0,73-1,23$ & & \\
\hline \multicolumn{7}{|c|}{...tenha outras coisas } \\
\hline \multicolumn{7}{|c|}{ interessantes para fazer } \\
\hline Discordo & 486 & 43,8 & 1,00 & & & \\
\hline Concordo & 623 & 56,2 & 1,03 & $0,79-1,34$ & & \\
\hline \multicolumn{7}{|c|}{$\begin{array}{l}\text {...não tenha ninguém para ir } \\
\text { comigo }\end{array}$} \\
\hline Discordo & 309 & 27,9 & 1,00 & & 1,00 & \\
\hline Concordo & 800 & 72,1 & 0,71 & $0,54-0,94$ & 0,73 & $0,56-0,95 * *$ \\
\hline \multicolumn{7}{|c|}{...amigos convidem para fazer } \\
\hline \multicolumn{7}{|l|}{ outras coisas } \\
\hline Discordo & 543 & 49,0 & 1,00 & & & \\
\hline Concordo & 566 & 51,0 & 1,01 & $0,78-1,32$ & & \\
\hline \multicolumn{7}{|c|}{...pudesse assistir TV, jogar } \\
\hline \multicolumn{7}{|c|}{ videogames, usar computador } \\
\hline Discordo & 792 & 71,4 & 1,00 & & & \\
\hline Concordo & 317 & 28,6 & 1,17 & $0,87-1,58$ & & \\
\hline \multirow{2}{*}{\multicolumn{7}{|c|}{$\begin{array}{l}\text {...esteja sem vontade, } \\
\text { desmotivado }\end{array}$}} \\
\hline & & & & & & \\
\hline Discordo & 861 & 77,6 & 1,00 & & 1,00 & \\
\hline Concordo & 248 & 22,4 & 0,74 & $0,53-1,03$ & 0,81 & $0,60-1,10$ \\
\hline \multirow{2}{*}{\multicolumn{7}{|c|}{$\begin{array}{l}\text {...tenha que pagar taxa, } \\
\text { mensalidade }\end{array}$}} \\
\hline & & & & & & \\
\hline Discordo & 718 & 64,7 & 1,00 & & 1,00 & \\
\hline Concordo & 391 & 35,3 & 1,25 & $0,95-1,65$ & 1,30 & $1,00-1,69 * *$ \\
\hline \multicolumn{7}{|c|}{...ache que não tenha habilidade } \\
\hline Discordo & 575 & 51,8 & 1,00 & & & \\
\hline Concordo & 534 & 48,2 & 1,07 & $0,81-1,41$ & & \\
\hline \multicolumn{7}{|c|}{...não tenham locais próximos a } \\
\hline \multicolumn{7}{|c|}{ minha casa } \\
\hline Discordo & 502 & 45,3 & 1,00 & & & \\
\hline Concordo & 607 & 54,7 & 1,15 & $0,88-1,52$ & & \\
\hline \multicolumn{7}{|c|}{...não tenha ninguém para } \\
\hline \multicolumn{7}{|l|}{ orientar } \\
\hline Discordo & 469 & 42,3 & 1,00 & & 1,00 & \\
\hline Concordo & 640 & 57,7 & 0,83 & 0,64-1,09 & 0,88 & $0,68-1,13$ \\
\hline \multicolumn{7}{|c|}{ Escore da autoeficácia } \\
\hline Baixa & 558 & 50,3 & 1,00 & & & \\
\hline Elevada & 551 & 49,7 & 0,78 & $0,76-1,23$ & & \\
\hline
\end{tabular}

IC95\%: intervalo de 95\% de confiança; RC: razão de chance.

* As variáveis com $p<0,20$ foram inseridas novamente no modelo;

$* * p<0,05$; 


\section{Figura 1}

Interação dos fatores sociodemográficos na associação dos fatores psicossociais com o transporte ativo para meninos.

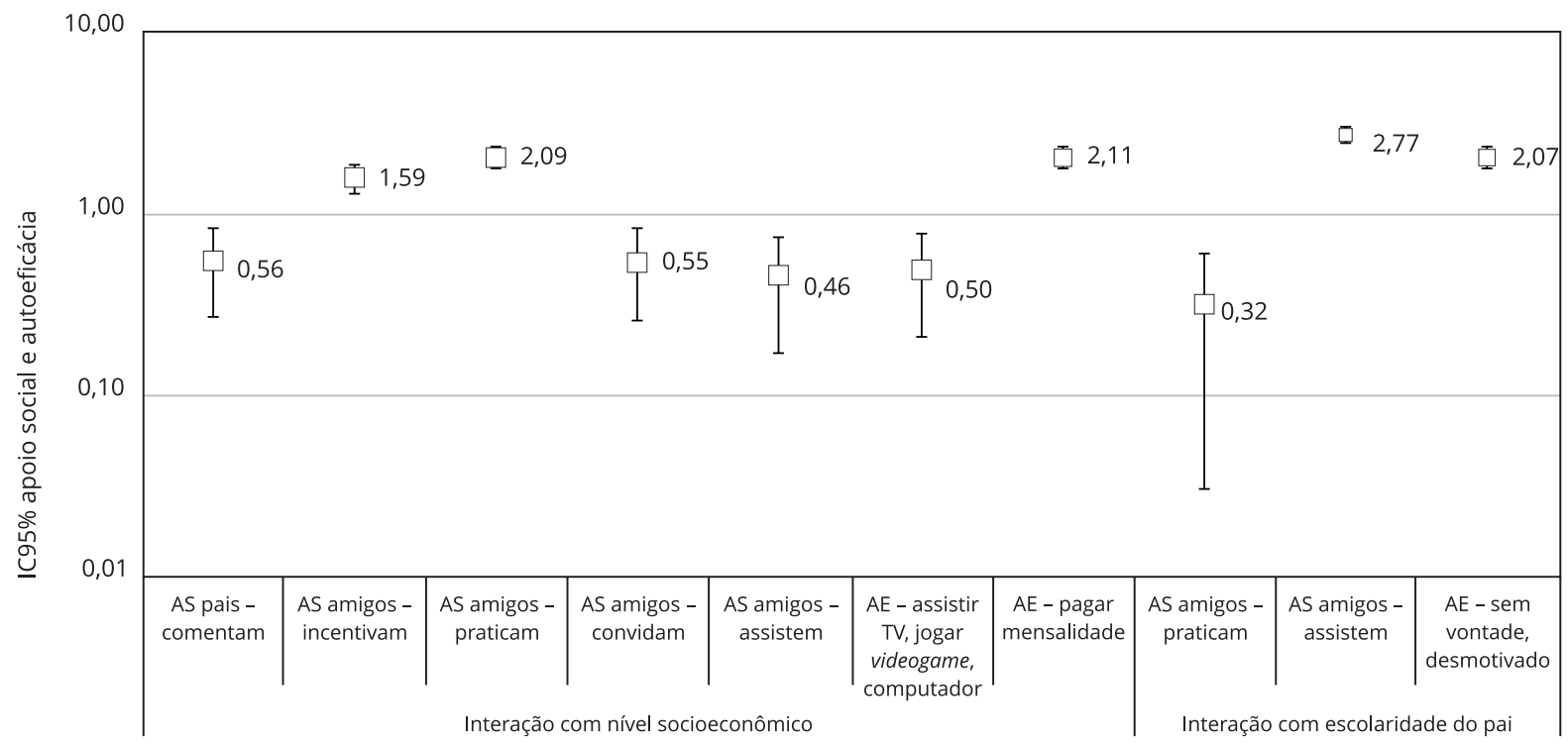

AE: autoeficácia; AS: apoio social; IC95\%: intervalo de 95\% de confiança.

Nota: ao todo, 92 interações foram testadas (as quatro variáveis sociodemográficas foram testadas com as 12 variáveis da escala de apoio social e as 11 variáveis da escala de autoeficácia). As interações significativas $(p<0,05)$ foram apresentadas na figura. 


\section{Figura 2}

Interação dos fatores sociodemográficos na associação dos fatores psicossociais com o transporte ativo para meninas.

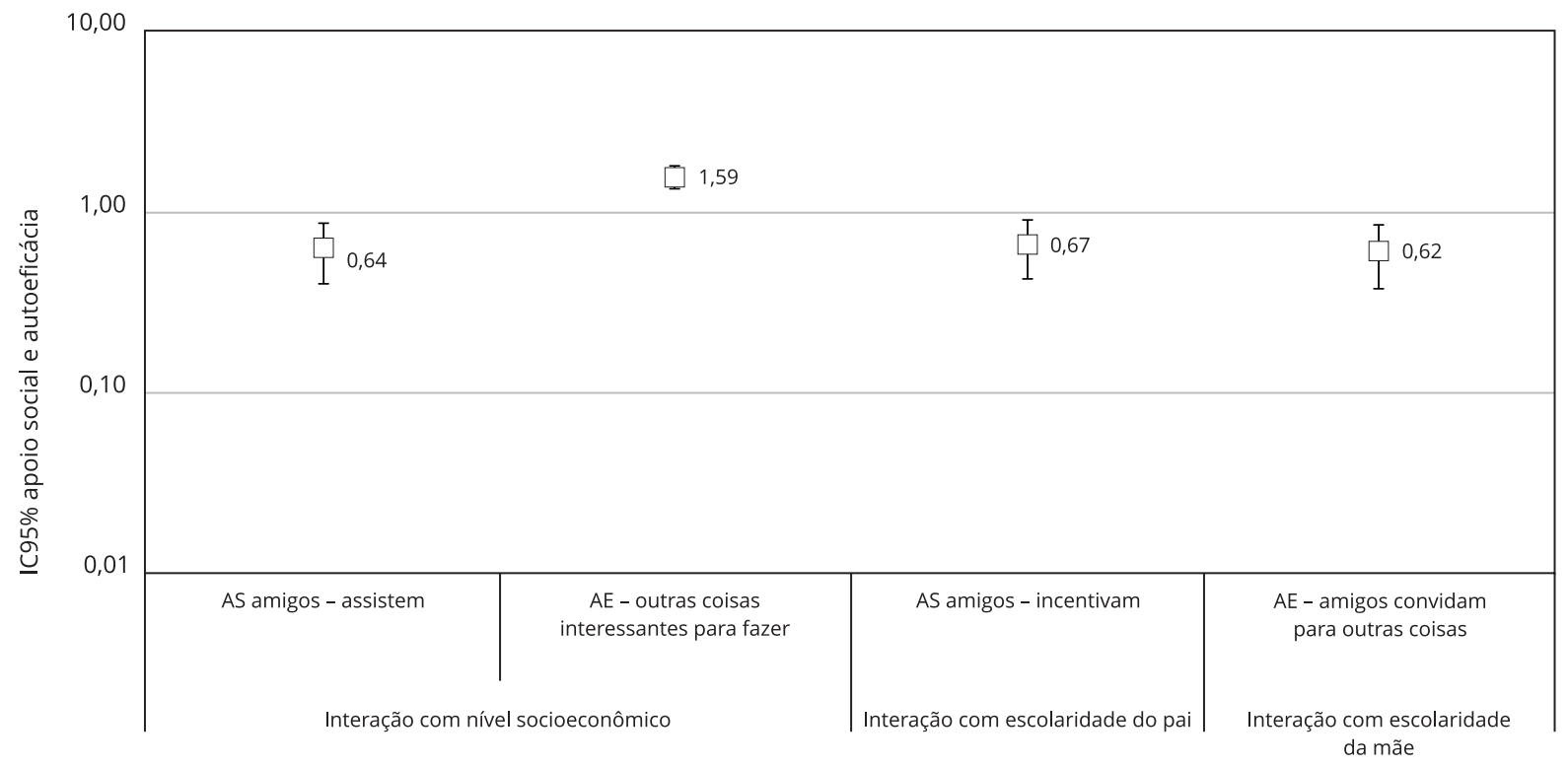

AE: autoeficácia; AS: apoio social; IC95\%: intervalo de 95\% de confiança.

Nota: ao todo, 92 interações foram testadas (as quatro variáveis sociodemográficas foram testadas com as 12 variáveis da escala de apoio social e as 11 variáveis da escala de autoeficácia). As interações significativas $(p<0,05)$ foram apresentadas na figura.

\section{Discussão}

O transporte ativo para a escola é uma maneira eficaz de incorporar atividade física às atividades diárias e aumentar os níveis gerais de atividade física 14. Em especial, na transição do final da adolescência para a idade adulta jovem, período crítico associado à diminuição da atividade física 1,4, o transporte ativo para a escola demonstra ser uma importante ferramenta para maximizar os níveis de atividade física em adolescentes 14,15,16,17,18,19. Ademais, a literatura apresenta que os fatores psicossociais estão associados com o transporte ativo para a escola 14,15,16,17,18,19. Porém, essa associação pode ser distinta, dependendo de sexo, idade, escolaridade de pais e nível socioeconômico, contexto cultural e geográfico. Isso pode resultar em um possível efeito moderador dos fatores sociodemográficos, o que até o momento foi pouco investigado.

Com base nessa explanação, o presente estudo buscou investigar a interação dos fatores sociodemográficos (idade, escolaridade dos pais, nível socioeconômico). Foi identificado que, para meninos, a associação do apoio social dos pais com o transporte ativo para a escola foi moderado pelo nível socioeconômico. Ainda, o apoio social dos amigos e a autoeficácia foram moderadas pelo nível socioeconômico e escolaridade dos pais. Para meninas, o apoio social de amigos foi moderado pelo nível socioeconômico e escolaridade de pai e mãe. A autoeficácia foi moderada somente pelo nível socioeconômico. A idade não apresentou valores significativos nas análises de interação, talvez devido ao fato de os adolescentes serem todos mais velhos (15 a 17 anos).

Em um estudo realizado no Brasil 32, com adolescentes de escolas públicas e privadas de João Pessoa (Paraíba), o sexo e a classe econômica moderaram a associação entre os fatores psicossociais e a atividade física, concluindo que possíveis influências da percepção de apoio social e autoeficácia com níveis de atividade física variam conforme as características sociodemográficas dos adolescentes 32,33,34,35. Alguns estudos de barreiras também mencionam que adolescentes percebem barreiras para 
atividade física (nos diferentes contextos) de maneira diferente em relação ao sexo e nível socioeconômico 36,37. Isso demonstra a importância de analisar as investigações sobre atividade física separadas por sexo e, ainda, o papel do nível socioeconômico.

Os resultados das interações envolvendo transporte ativo para a escola e variáveis psicossociais foram geralmente consistentes, quando comparados à literatura que apresenta que escolaridade dos pais e nível socioeconômico influenciam os diferentes contextos da atividade física (transporte, lazer) $32,33,34,35,38$. A literatura apresenta que adolescentes mais novos têm mais chances de realizar transporte ativo para a escola, quando comparados aos mais velhos, assim como adolescentes que pertencem a um nível socioeconômico mais baixo e que possuem pais com baixa escolaridade 32,33,34,35.

Escolaridade alta e nível socioeconômico alto não foram associados com o transporte ativo para a escola em outros estudos com adolescentes 32,33,34,35. Isso demonstra similaridade nos resultados do presente estudo, uma vez que as análises de interação que apresentaram associação negativa foram as análises com maiores prevalências de pais com escolaridade e nível socioeconômico alto. Em contrapartida, as análises de interação que apresentaram associação positiva tiveram mais prevalências de pais com escolaridade e nível socioeconômico baixo. Esses dados contribuem para uma melhor compreensão do tema.

As razões para que esse efeito moderador ocorra não são claras, mas é possível que a escolaridade dos pais e o nível socioeconômico mais elevado considerem outros constrangimentos ao transporte ativo para a escola, tais como os horários ou a percepção de segurança 14,15,16,17,18,19, independente do suporte para a prática de atividade física em outros contextos 38,39. A literatura apresenta que, quando pais e amigos fornecem mais apoio social, os adolescentes passam a referir maiores níveis de atividade física 39. O presente estudo colabora com essa linha ao investigar o contexto transporte, visto que esse contexto pode ser uma forma de promover atividade física entre adolescentes. Em acréscimo, a mesma revisão sistemática 39 apresenta que, na adolescência, o apoio social de amigos é reportado com mais frequência quando comparado ao apoio social dos pais. Na análise de interação do presente estudo, a escala de apoio social dos amigos apresentou um número maior de variáveis significativas, quando comparada às escalas de apoio social de pais e autoeficácia. Isso demonstra que, mesmo com a inclusão das variáveis nível socioeconômico e escolaridade, o apoio social dos amigos demonstrou ter uma relação mais presente quando comparado às outras variáveis psicossociais.

Alguns pontos devem ser considerados na interpretação dos resultados deste estudo. O delineamento transversal limita a adequada interpretação da relação causal entre as variáveis. Amostra formada apenas com estudantes da rede pública impossibilita a extrapolação dos resultados para classes mais elevadas. Medidas relatadas também dependem da veracidade e do poder recordatório das respostas do entrevistado. Entretanto, a amostra representativa e as análises estatísticas asseguram uma interpretação dos dados para grandes populações de escolas da rede pública, um ponto importante no domínio de intervenções relacionadas à saúde pública e preventiva. Ademais, torna-se um dos primeiros estudos que investigou a interação dos fatores sociodemográficos na relação entre fatores psicossociais e transporte ativo para a escola em uma amostra representativa de adolescentes.

São necessários investimentos para melhorar a segurança pública, assim como a implantação e a melhoria de estruturas, próximas às escolas, que aumentem a segurança no trânsito, como ciclovias/ ciclofaixas e redutores de velocidade dos veículos, para que adolescentes possam usar meios de transporte ativos (caminhada/bicicleta/skate). Além disso, campanhas de conscientização dos pais sobre a importância do apoio social para a prática de atividade física em todos os contextos podem ser implementadas nas escolas. Futuros estudos de intervenção poderiam analisar o custo-efetividade que as intervenções ambientais (segurança, estrutura para bicicletas/skates, calçadas e demais) proporcionam no uso de meios de transporte ativos (caminhada, bicicleta, skate) para a escola.

\section{Conclusão}

Os resultados indicam que os fatores sociodemográficos (escolaridade dos pais e nível socioeconômico) moderam a associação dos fatores psicossociais (apoio social dos pais e amigos e autoeficácia) com o transporte ativo para a escola. As análises de interação demonstraram que escolaridade e nível socioeconômico alto estão associados negativamente ao transporte ativo para a escola, e nível socioe- 
conômico baixo e escolaridade baixa estão associados positivamente. Para meninos, um número maior de variáveis das escalas de apoio social e autoeficácia foi significativo (na análise de interação) quando comparado ao das meninas. A escala de apoio social dos amigos apresentou um número maior de variáveis significativas, quando comparada às escalas de apoio social de pais e autoeficácia. Isso demonstra que, mesmo com a inclusão das variáveis nível socioeconômico e escolaridade, o apoio social dos amigos demonstrou ter uma relação mais presente quando comparado a outras variáveis psicossociais.

\section{Colaboradores}

E. M. Camargo foi responsável por todos os aspectos na elaboração do trabalho. M. P. M. Santos e A. G. P. Ribeiro contribuíram na redação do manuscrito. J. Mota e W. Campos contribuíram na revisão crítica do manuscrito. Todos os autores revisaram e aprovaram a versão final para publicação.

\section{Informações adicionais}

ORCID: Edina Maria de Camargo (0000-00032127-2606); Maria Paula Maia Santos (0000-00022182-9841); Anelize Gabriele Peressute Ribeiro (0000-0003-3835-9396); Jorge Mota (0000-00017571-9181); Wagner de Campos (0000-0003-39791017).

\section{Agradecimentos}

Agradecemos a todas as escolas visitadas e sua ótima recepção à equipe de coleta de dados, aos estudantes que se voluntariaram a participar do estudo, bem como seus respectivos responsáveis pela autorização aos mesmos.

\section{Referências}

1. Abarca-Gómez L, Abdeen ZA, Hamid ZA, Abu-Rmeileh NM, Acosta-Cazares B, Acuin C, et al. Worldwide trends in body-mass index, underweight, overweight, and obesity from 1975 to 2016: a pooled analysis of 2416 population-based measurement studies in 128.9 million children, adolescents, and adults. Lancet 2017; 390:2627-42.

2. Ding D, Lawson KD, Kolbe-Alexander TL, Finkelstein EA, Katzmarzyk PT, van Mechelen $\mathrm{W}$, et al. The economic burden of physical inactivity: a global analysis of major non-communicable diseases. Lancet 2016; 388:1311-24.

3. Blair SN. Physical inactivity: the biggest public health problem of the 21st century. Br J Sports Med 2009; 43:1-2.

4. Sallis JF, Bull F, Guthold R, Heath GW, Inoue $\mathrm{S}$, Kelly P, et al. Progress in physical activity over the Olympic quadrennium. Lancet 2016; 388:1325-36.

5. Hoehner CM, Soares J, Parra Perez D, Ribeiro IC, Joshu CE, Pratt M, et al. Physical activity interventions in Latin America. Am J Prev Med 2008; 34:224-33.

6. Ruiz JR, Ortega FB, Martinez-Gomez D, Labayen I, Moreno LA, De Bourdeaudhuij I, et al. Objectively measured physical activity and sedentary time in European adolescents: the HELENA Study. Am J Epidemiol 2011; 174:173-84.

7. World Health Organization. Global recommendations on physical activity for health. Geneva: World Health Organization; 2010.

8. Roberts C, Freeman J, Samdal O, Schnohr CW, de Looze ME, Nic Gabhainn S, et al. The Health Behaviour in School aged Children (HBSC) study: methodological developments and current tensions. Int J Public Health 2009; 54 Suppl 2:S140-50.

9. Ruiz E, Ávila JM, Castillo A, Valero T, del Pozo S, Rodriguez P, et al. The ANIBES study on energy balance in Spain: design, protocol and methodology. Nutrients 2015; 7:970-98.

10. Baptista, F, Santos DA, Silva AM, Mota J, Santos R, Vale S, et al. Prevalence of the Portuguese population attaining sufficient physical activity. Med Sci Sports Exerc 2012; 44:46673.

11. Reis AAC, Malta DC, Furtado LAC. Desafios para as políticas públicas voltadas à adolescência e juventude a partir da Pesquisa Nacional de Saúde do Escolar (Pense). Ciênc Saúde Colet $2018 ; 23: 2879-90$. 
12. Nardo N, Silva DAS, de Moraes Ferrari GL, Petroski EL, Pacheco RL, Martins PC, et al. Results from Brazil's 2016 Report Card on Physical Activity for Children and Youth. J Phys Act Health 2016; 13 (11 Suppl 2):10409.

13. Cureau FV, da Silva TLN, Bloch KV, Fujimori E, Belfort DR, de Carvalho KMB, et al. ERICA: leisure-time physical inactivity in Brazilian adolescents. Rev Saúde Pública 2016; 50 Suppl 1:4s.

14. Verhoeven H, Simons D, Van Dyck D, Van Cauwenberg J, Clarys P, De Bourdeaudhuij I, et al. Psychosocial and environmental correlates of walking, cycling, public transport and passive transport to various destinations in flemish older adolescents. PLoS One 2016; 11:e0147128.

15. Dalton MA, Longacre MR, Drake KM, Gibson L, Adachi-Mejia AM, Swain K, et al. Built environment predictors of active travel to school among rural adolescents. Am J Prev Med 2011; 40:312-9.

16. Babey SH, Hastert TA, Huang W, Brown ER. Sociodemographic, family, and environmental factors associated with active commuting to school among US adolescents. J Public Health Policy 2009; 30 Suppl 1:S203-20.

17. Nelson NM, Foley E, O'Gorman DJ, Moyna NM, Woods CB. Active commuting to school: how far is too far? Int J Behav Nutr Phys Act 2008; 5:1.

18. van Dyck D, De Bourdeaudhuij I, Cardon G, Deforche B. Criterion distances and correlates of active transportation to school in Belgian older adolescents. Int J Behav Nutr Phys Act 2010; 7:87.

19. Faulkner GE, Buliung RN, Flora PK, Fusco C. Active school transport, physical activity levels and body weight of children and youth: a systematic review. Prev Med 2009; 48:3-8.

20. Bauman AE, Reis RS, Sallis JF, Wells JC, Loos RJ, Martin BW. Correlates of physical activity: why are some people physically active and others not? Lancet 2012; 380:258-71.

21. Silva KS, Pizarro AN, Garcia, LMT, Mota J, Santos MP. Which social support and psychological factors are associated to active commuting to school? Prev Med 2014; 63:20-3.

22. Bauman AE, Sallis JF, Dzewaltowski DA, Owen N. Toward a better understanding of the influences on physical activity: the role of determinants, correlates, causal variables, mediators, moderators, and confounders. Am J Prev Med 2002; 23(2 Suppl):5-14.

23. Fermino RC, Rech CR, Hino AA, Rodriguez Anez CR, Reis RS. Physical activity and associated factors in highschool adolescents in Southern Brazil. Rev Saúde Pública 2010; 44:986-95.

24. Barbosa Filho VC, Campos W, Bozza R, Lopes AS. The prevalence and correlates of behavioral risk factors for cardiovascular health among Southern Brazil adolescents: a cross-sectional study. BMC Pediatr 2012; 12:130.

25. Farias Júnior JC, Mendonça G, Florindo AA, Barros MVG. Reliability and validity of a physical activity social support assessment scale in adolescents - ASAFA Scale. Rev Bras Epidemiol 2014; 17:355-70.
26. Prado CV, Lima AV, Fermino RC, Añez CRR, Reis RS. Apoio social e prática de atividade física em adolescentes da rede pública de ensino: qual a importância da família e dos amigos? Cad Saúde Pública 2014; 30:827-38.

27. Farias Junior JC, Lopes AS, Reis RS, Nascimento JV, Borgatto AF, Hallal PC. Development and validation of a questionnaire measuring factor associated with physical activity in adolescents. Rev Bras Saúde Mater Infant 2011; 11:301-12.

28. Souza CA, Rech CR, Sarabia TT, Añez CRR, Reis RS. Autoeficácia e atividade física em adolescentes de Curitiba, Paraná, Brasil. Cad Saúde Pública 2013; 29:2039-48.

29. Van Dyck D, Cardon G, Deforche B, De Bourdeaudhuij I. Lower neighbourhood walkability and longer distance to school are related to physical activity in Belgian adolescents. Prev Med 2009; 48:516-18.

30. Wong BY-M, Faulkner G, Buliung R. GIS measured environmental correlates of active school transport: a systematic review of 14 studies. Int J Behav Nutr Phys Act 2011; 8:39.

31. Becker LA, Fermino RC, Lima AV, Rech CR, Añez C, Reis R. Perceived barriers for acrive commuting to school among adolescents from Cruritiba, Brasil. Rev Bras Ativ Fís Saúde 2017; 22:24-34.

32. Cheng LA, Mendonça G, Farias Junior, JC. A associação entre fatores psicossociais e atividade física em adolescentes é moderada por fatores sociodemográficos? Rev Bras Ativ Fís Saúde 2016; 21:297-306.

33. Rech RR, Risa CO, Avrela PR, Halpern R, Constanzi CB, Bergmann ML, et al. Fatores associados ao deslocamento ativo em escolares. Rev Bras Ativ Fis Saúde 2013; 18:332-38.

34. Farias Junior JC, Reis RS, Hallal PC. Physical activity, psychosocial and perceived environmental factors in adolescentes from Northeast Brasil. Cad Saúde Pública 2014; 30:941-51.

35. Silva KS, Lopes AS, Silva FM. Atividade física no deslocamento à escola e no tempo livre em crianças e adolescentes da cidade de João Pessoa, PB, Brasil. Rev Bras Ciênc Mov 2007; 15:61-70.

36. Santos MS, Hino AAF, Reis RS, Rodriguez -Añez CR. Prevalência de barreiras para a prática de atividade física em adolescentes. Rev Bras Epidemiol 2010; 13:94-104.

37. Rech CR, Camargo EM, Araujo PAB, Loch MR, Reis, RS. Perceived barriers to leisuretime physical activity in the brazilian population. Rev Bras Med Esporte 2018; 24:303-9.

38. Cheng LA, Mendonça G, Farias Júnior JC. Physical activity in adolescents: analysis of the social influence of parents and friends. J Pedi$\operatorname{atr}$ (Rio J.) 2014; 90:35-41.

39. Mendonça G, Cheng LA, Melo EN, Farias Junior JC. Physical activity and social support in adolescents: a systematic review. Health Educ Res 2014; 29:822-39. 


\section{Abstract}

The study aimed to analyze the interaction of sociodemographic factors in the association between psychosocial factors and active commuting to school. This was a cross-sectional study in 2018 with a sample of 1,984 students. The target sociodemographic factors were sex, age, parents' schooling, and socioeconomic status. Social support and self-efficacy were measured with questionnaires. Active commuting to school was defined as walking, bicycling, or skateboarding to and from school. Binary logistic regression was used to verify the association between psychosocial factors and active commuting to school. Then, interaction terms with the sociodemographic variables (age, father's schooling, mother's schooling, socioeconomic status) were included in the relationship between psychosocial factors and active commuting to school. The sample consisted of 1,984 adolescents (55.9\% females). Of these, 748 (37.7\% of the sample) reported active commuting to school during a normal week. Analyses of interaction showed that high levels of schooling and high socioeconomic status were negatively associated with active commuting to school. Low socioeconomic status and low schooling were positively associated with such commuting. The peer social support scale showed more significant variables than the other scales. The results indicate that sociodemographic factors (parents' schooling and socioeconomic status) moderate the association between psychosocial factors and active commuting to school.

\section{Motor Activity; Adolescent; Social Support; Self Efficacy}

\section{Resumen}

El objetivo fue investigar la interacción de los factores sociodemográficos en la asociación de los factores psicosociales con el transporte activo hacia la escuela. Estudio transversal, llevado a cabo en 2018, con una muestra de 1.984 estudiantes. Los factores sociodemográficos incluidos fueron: sexo, edad, escolaridad de los padres y nivel socioeconómico. El apoyo social y la autoeficacia fueron medidos por medio de cuestionarios. Se consideró transporte activo hacia la escuela: caminar, pedalear o usar monopatín. La regresión logística binaria se utilizó para verificar la asociación de los factores psicosociales con el transporte activo a la escuela. Después, los términos de interacción con las variables sociodemográficas (edad, escolaridad del padre, escolaridad de la madre, nivel socioeconómico) se incluyeron en la relación de los factores psicosociales con el transporte activo hacia la escuela. La muestra estaba compuesta por 1.984 adolescentes (55,9\% de sexo femenino). De ellos, 748 (37,7\% de la muestra) informaron utilizar transporte activo hacia la escuela durante una semana normal. Los análisis de interacción demostraron que escolaridad y nivel socioeconómico alto están asociados negativamente con el transporte activo hacia la escuela. El nivel socioeconómico bajo y la escolaridad baja están asociados positivamente. La escala de apoyo social de los amigos presentó un número mayor de variables significativas, cuando se compara con las demás escalas. Los resultados indican que los factores sociodemográficos (escolaridad de los padres y nivel socioeconómico) moderan la asociación de los factores psicosociales con el transporte activo hacia la escuela.

Actividad Motora; Adolescente; Apoyo Social; Autoeficacia
Recebido em 31/Mai/2019

Versão final reapresentada em 28/Out/2019

Aprovado em 11/Nov/2019 